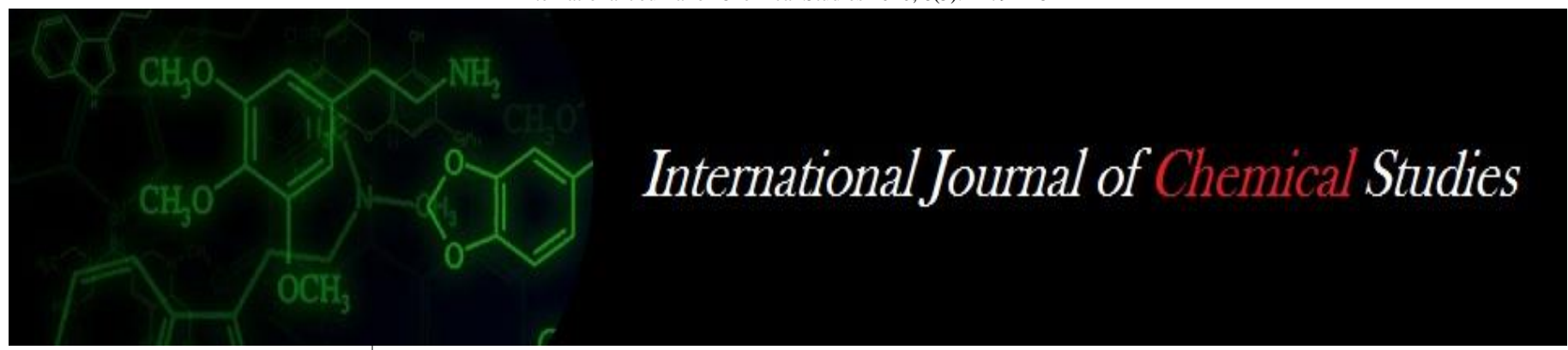

P-ISSN: 2349-8528

E-ISSN: 2321-4902

www.chemijournal.com

IJCS 2020; 8(5): 1279-1282

(C) 2020 IJCS

Received: 28-06-2020

Accepted: 12-08-2020

Vipul Y Patel

Department of Agronomy,

BACA, AAU, Anand, Gujarat,

India

\section{BD Patel}

Agronomist \& PI, AICRP Weed

Management, BACA, AAU,

Anand, Gujarat, India

\section{VJ Patel}

Associate Professor, Sheth M. C. Polytechnic in Agriculture,

AAU, Anand, Gujarat, India

\section{DD Chaudhari}

Jr. Agronomist, AICRP Weed

Management, BACA, AAU,

Anand, Gujarat, India

\section{NJ Chaudhari}

Assistant Professor (Agronomy) Department of Ag. Meteorology, AAU, Anand, Gujarat, India

Corresponding Author:

Vipul Y Patel

Department of Agronomy,

BACA, AAU, Anand, Gujarat,

India

\section{Effect of herbicide combinations on growth, yield and nutrient uptake by weeds in irrigated wheat}

\author{
Vipul Y Patel, BD Patel, VJ Patel, DD Chaudhari and NJ Chaudhari
}

DOI: https://doi.org/10.22271/chemi.2020.v8.i5r.10478

\begin{abstract}
An experiment was carried out for two consecutive rabi season of the year 2017-18 and 2018-19 to investigate the "Effect of herbicide combinations on growth, yield and nutrient uptake by weeds in irrigated wheat. Results showed that post emergence application of pre-mix herbicides sulfosulfuron + metsulfuron $30+2 \mathrm{~g} / \mathrm{ha}$, clodinafop + metsulfuron $60+4 \mathrm{~g} / \mathrm{ha}$ and mesosulfuron + iodosulfuron $12+2.4$ $\mathrm{g} / \mathrm{ha}$ recorded significantly lower density and dry biomass of weeds, improve the promising yield attributing viz., number of effective tillers $/ \mathrm{m}^{2}$, number of total tillers $/ \mathrm{m}^{2}$, tiller conversion index and grain yield as well as straw yield with minimum nutrient uptake by weeds than other treatments. Sequential application of pendimethalin (PE) $500 \mathrm{~g} / \mathrm{ha} f b$ sulfosulfuron (PoE) $18 \mathrm{~g} / \mathrm{ha}$, twice hand weeding at 20 and 40 DAS and tank mix formulation of sulfosulfuron + 2,4-D (SS) (PoE) $25+250 \mathrm{~g} / \mathrm{ha}$ was stood second best treatment during both the years.
\end{abstract}

Keywords: Premix, tank mix, monocot, dicot, tiller, TCI, uptake

\section{Introduction}

In India wheat is second most important staple food crop after rice. The major wheat growing states in India are Uttar Pradesh, Punjab, Madhya Pradesh, Haryana, Rajasthan, Bihar and Gujarat. The average productivity of wheat in India was to the tune of $3216 \mathrm{~kg} / \mathrm{ha}$. In Gujarat, the average productivity of wheat was $2751 \mathrm{~kg} / \mathrm{ha}$ (Anon., 2018) ${ }^{[2]}$ which is lower than northern states like Punjab and Haryana as the shorter duration of winter season in Gujarat does not provide much congenial climatic condition for its growth. Another major factor responsible for low productivity in wheat is weed infestation because it competes with crop for all necessary resources viz., soil moisture, nutrient, sunlight and space. Weed infestation also leads to deteriorate quality of crop product and hazardous to human and animal health also. Improper weed management or Uncontrolled weeds are reported to cause up to $66 \%$ reduction in wheat grain yield (Angiras et al. 2008) ${ }^{[1]}$. As the crop is badly infested with narrow and broad leaved weeds like Parthenium hysterophorus, Portulaca oleracea, Euphorbia mollis, Amaranthus viridis, Convolvulus arvensis, Commelina benghalensis, Chenopodium album, Cyperus rotundus and Sonchus arvensis etc., those are appears regularly because of intensive cultivation with frequent irrigation and dominance of monotony cropping system. However, continuous use of same herbicide with improper dose and faulty application can leads to develop weed resistance to herbicides. Avoidance of weed resistance to herbicide and effective control of weed flora is possible through using herbicide combination because it require lower dose, broad spectrum and increase the activity on targeted weed without causing any crop injury and also cost effective and time saving as compared to hand weeding and mechanical method. Looking to this the experiment was planned.

\section{Materials and methods}

The experiment was conducted at the farm of AICRP-Weed Management, B. A. College of Agriculture, Anand Agricultural University, Anand (Gujarat) during rabi-summer season of the year 2017-18 and 2018-19 on loamy sand soil. The experiment was laid out in randomized block design with three replication and tried different twelve treatment viz., $\mathrm{T}_{1}$ : Pendimethalin PE (750 g/ha), T $\mathrm{T}_{2}$ : Pendimethalin + metribuzin (Tank mix) PE $(500+175 \mathrm{~g} / \mathrm{ha}), \mathrm{T}_{3}$ : Pendimethalin PE (500 g/ha) $f b$ sulfosulfuron PoE (18 g/ha), T : Sulfosulfuron PoE (25 g/ha), $\mathrm{T}_{5}$ : Sulfosulfuron + 2,4-D (SS) (Tank mix) PoE $(25+250 \mathrm{~g} / \mathrm{ha}), \mathrm{T}_{6}$ : Sulfosulfuron + 
metsulfuron (Premix) PoE $(30+2 \mathrm{~g} / \mathrm{ha}), \mathrm{T}_{7}$ : Clodinafop PoE $(60 \mathrm{~g} / \mathrm{ha}), \mathrm{T}_{8}$ : Clodinafop + metsulfuron (Premix) PoE $(60+4$ g/ha), $\mathrm{T}_{9}$ : Metsulfuron methyl PoE (4 g/ha), $\mathrm{T}_{10}$ : Mesosulfuron + iodosulfuron (Premix) PoE $(12+2.4 \mathrm{~g} / \mathrm{ha})$, $\mathrm{T}_{11}$ : Hand weeding (20 and $40 \mathrm{DAS}$ ) and $\mathrm{T}_{12}$ : Un-weeded control and replicated three times in rabi season during the years 2017-18 and 2018-19. Wheat cv. "Gujarat wheat 451" seeds were treated with vitavax $3 \mathrm{~g} / \mathrm{kg}$ seed, for the control of seed and soil borne diseases and also as plant growth stimulant before sowing. The recommended seed rate of wheat $120 \mathrm{~kg} / \mathrm{ha}$ was sown keeping the distance of $22.5 \mathrm{~cm}$ row spacing by manually in previously open furrows with the help of kudali. Later the seeds were covered manually. The crop was sown during two consecutive rabi season on $18^{\text {th }}$ November, 2017 and $27^{\text {th }}$ November, 2018. Pre-emergence application of herbicides was done one day after sowing while post-emergence herbicides were applied at 25-30 days after sowing with knapsack sprayer fitted with flat fan nozzle by using 500 litre of water/ha. At the maturity of crop, border lines were harvested first and were removed from the experimental area. Then the net plot area was harvested separately.

Weed parameters measure taken randomly from $0.25 \mathrm{~m}^{2}$ quadrant from net plot area from each treatment and converted into $\mathrm{m}^{2}$ area. The monocot and dicot weeds were separated and counted separately from each plot. The mean data are used for analysis purpose. Data on weed density and weed dry weight were analysed through square root transformation. Yield attributes character number of tillers per meter square was count from net plot area.

\section{Result and discussion \\ Weed flora}

Weedy check plot was heavily infested with monocot weeds like Phalaris minor, Avena fatua and Setaria tomentosaas well as dicot weeds viz., Chenopodium murale, Chenopodium album, Melilotus alba and Asphodelus tenuifolius. The highest relative density of 59.82 per cent was recorded under monocot weeds while dicot weeds constituted about 40.18 per cent of the total weed population on two years mean basis.

\section{Weed density and dry weight}

Application of pre-mix herbicide sulfosulfuron + metsulfuron $(\mathrm{PoE}) 30+2 \mathrm{~g} / \mathrm{ha}\left(\mathrm{T}_{6}\right)$, clodinafop + metsulfuron $(\mathrm{PoE}) 60+$ $4 \mathrm{~g} / \mathrm{ha}\left(\mathrm{T}_{8}\right)$ and mesosulfuron + iodosulfuron $(\mathrm{PoE}) 12+2.4$ $\mathrm{g} / \mathrm{ha}\left(\mathrm{T}_{10}\right)$ provided complete control of grassy and broad-leaf weeds so, weed dry matters could not recorded during both the experimentation year (Tables 1 and 2). Similarly, Punia et al., (2008) ${ }^{[10]}$ also observed that application of ready-mix formulation of clodinafop $15 \%+$ metsulfuron $1 \%$ at $60 \mathrm{~g} / \mathrm{ha}$ $+4 \mathrm{~g} / \mathrm{ha}$ as post emergence recorded 97 and 100 per cent control of weeds in case of grassy and broad-leaf weeds, respectively. Tank mix application of sulfosulfuron $+2,4-\mathrm{D}$ (SS) $(\mathrm{PoE}) 25+250 \mathrm{~g} / \mathrm{ha}\left(\mathrm{T}_{5}\right)$ was found effective against dicot weeds and they provided cent per cent broad-leaf weeds during both the year of experimentation. Tank mix/pre-mix application of herbicide effectively reduced dry weight of weeds due to combination exhibit properties of both foliar and soil activity that inhibits cell division in shoots and roots and growth by inhibiting plant enzyme acetolactase synthase thereby, blocking branches chain of amino acid biosynthesis and hence, the plant suffers. Due to this, phloem transport of the plant is hampered. A secondary effect is stunted growth on account of cessation of cell division and slow plant death. Contrary to the alone application of either of the herbicide was not found effective to control all sort of the weeds in the entire crop year (Lekhchand ${ }^{[5]}$, Chaudhari et al. $2017^{[4]}$ and Meena et al., 2019) ${ }^{[6]}$.

Sequential application of pendimethalin (PE) $500 \mathrm{~g} / \mathrm{ha} \mathrm{fb}$ sulfosulfuron (PoE) $18 \mathrm{~g} / \mathrm{ha}$, twice hand weeding at 20 and 40 DAS, sulfosulfuron + 2,4-D (SS) (PoE) $25+250 \mathrm{~g} / \mathrm{ha}$ and sulfosulfuron (PoE) $25 \mathrm{~g} / \mathrm{ha}$ was at par with pre-mix herbicides treatment in case of monocot and dicot weed density as well as weed dry weight. Sole application of clodinafop was impressively manage the grassy weeds but failed to control dicot weeds while sole application of metsulfuron methyl (PoE) $4 \mathrm{~g} / \mathrm{ha}\left(\mathrm{T}_{9}\right)$ was much effective against dicot weeds and they provide complete control of broad-leaf weeds during both the year of experimentation. Similar lines of results are in conformity with the finding of Malik et al. (2013) ${ }^{[8]}$.

Table 1: Density of monocot and dicot weeds as influenced by various weed management practices

\begin{tabular}{|c|c|c|c|c|c|c|c|c|}
\hline \multirow{3}{*}{$\begin{array}{l}\text { Treat } \\
\text { ment }\end{array}$} & \multicolumn{4}{|c|}{ Density of monocot weed $\left(\mathrm{no} . / \mathrm{m}^{2}\right)$} & \multicolumn{4}{|c|}{ Density of dicot weed $\left(\mathrm{no} / \mathrm{m}^{2}\right)$} \\
\hline & \multicolumn{2}{|c|}{ at 80 DAS } & \multicolumn{2}{|c|}{ at Harvest } & \multicolumn{2}{|c|}{ at 80 DAS } & \multicolumn{2}{|c|}{ at Harvest } \\
\hline & 2017-18 & 2018-19 & 2017-18 & 2018-19 & 2017-18 & 2018-19 & 2017-18 & 2018-19 \\
\hline \multirow{2}{*}{$\mathrm{T}_{1}$} & $4.37^{\mathrm{c}}$ & $5.24^{\mathrm{c}}$ & $4.70^{c}$ & $5.35^{\mathrm{c}}$ & $3.40^{\mathrm{bc}}$ & $4.10^{b c}$ & $3.15^{\mathrm{cd}}$ & $3.58^{\mathrm{bc}}$ \\
\hline & $(18.67)$ & $(26.67)$ & $(21.33)$ & $(28.00)$ & $(10.67)$ & $(16.00)$ & $(9.33)$ & $(12.00)$ \\
\hline \multirow{2}{*}{$\mathrm{T}_{2}$} & $4.20^{\mathrm{cd}}$ & $4.44^{\mathrm{cd}}$ & $4.10^{c}$ & $5.02^{\mathrm{c}}$ & $3.20^{\mathrm{bc}}$ & $3.65^{\mathrm{cd}}$ & $2.75^{\mathrm{de}}$ & $3.78^{\mathrm{b}}$ \\
\hline & $(17.33)$ & $(20.00)$ & $(16.00)$ & $(25.33)$ & $(9.33)$ & $(13.33)$ & $(6.67)$ & $(13.33)$ \\
\hline \multirow{2}{*}{$\mathrm{T}_{3}$} & $2.95^{\mathrm{e}}$ & $3.20^{\mathrm{e}}$ & $2.24^{\mathrm{d}}$ & $2.49^{\mathrm{d}}$ & $2.75^{\mathrm{c}}$ & $3.00^{\mathrm{d}}$ & $2.24^{\mathrm{e}}$ & $2.75^{\mathrm{c}}$ \\
\hline & $(8.00)$ & $(9.33)$ & $(4.00)$ & $(5.33)$ & $(6.67)$ & $(8.00)$ & $(4.00)$ & $(6.67)$ \\
\hline \multirow{2}{*}{$\mathrm{T}_{4}$} & $3.40^{\text {cde }}$ & $3.78^{\mathrm{de}}$ & $3.00^{\mathrm{d}}$ & $3.20^{\mathrm{d}}$ & $3.73^{\mathrm{b}}$ & $4.72^{\mathrm{b}}$ & $3.78^{\mathrm{c}}$ & $4.17^{b}$ \\
\hline & $\begin{array}{l}(10.67) \\
\end{array}$ & (13.33) & $(8.00)$ & $(9.33)$ & (13.33) & (21.33) & (13.33) & (17.33) \\
\hline \multirow{2}{*}{$\mathrm{T}_{5}$} & $3.20^{\mathrm{de}}$ & $3.78^{\mathrm{de}}$ & $2.49^{\mathrm{d}}$ & $3.00^{\mathrm{d}}$ & $1.00^{\mathrm{d}}$ & $1.00^{\mathrm{e}}$ & $1.00^{\mathrm{f}}$ & $1.00^{\mathrm{d}}$ \\
\hline & $(9.33)$ & $\begin{array}{l}(13.33) \\
\end{array}$ & (5.33) & $(8.00)$ & $(0.00)$ & $\begin{array}{l}(0.00) \\
\end{array}$ & $(0.00)$ & $(0.00)$ \\
\hline \multirow{2}{*}{$\mathrm{T}_{6}$} & $1.00^{\mathrm{f}}$ & $1.00^{\mathrm{f}}$ & $1.00^{\mathrm{e}}$ & $1.00^{\mathrm{e}}$ & $1.00^{\mathrm{d}}$ & $1.00^{\mathrm{e}}$ & $1.00^{\mathrm{f}}$ & $1.00^{\mathrm{d}}$ \\
\hline & $(0.00)$ & $(0.00)$ & $(0.00)$ & $(0.00)$ & $(0.00)$ & $(0.00)$ & $(0.00)$ & $(0.00)$ \\
\hline \multirow{2}{*}{$\mathrm{T}_{7}$} & $2.49^{\mathrm{e}}$ & $1.00^{\mathrm{f}}$ & $2.24^{\mathrm{d}}$ & $1.00^{\mathrm{e}}$ & $7.36^{\mathrm{a}}$ & $8.05^{\mathrm{a}}$ & $7.45^{\mathrm{b}}$ & $8.46^{\mathrm{a}}$ \\
\hline & $(5.33)$ & $(0.00)$ & $(4.00)$ & $(0.00)$ & (53.33) & $(64.00)$ & (54.67) & $(70.67)$ \\
\hline \multirow{2}{*}{$\mathrm{T}_{8}$} & $1.00^{\mathrm{f}}$ & $1.00^{\mathrm{f}}$ & $1.00^{\mathrm{e}}$ & $1.00^{\mathrm{e}}$ & $1.00^{\mathrm{d}}$ & $1.00^{\mathrm{e}}$ & $1.00^{\mathrm{f}}$ & $1.00^{\mathrm{d}}$ \\
\hline & $(0.00)$ & $(0.00)$ & $(0.00)$ & $(0.00)$ & $(0.00)$ & $(0.00)$ & $(0.00)$ & $(0.00)$ \\
\hline \multirow{2}{*}{$\mathrm{T}_{9}$} & $6.58^{\mathrm{b}}$ & $7.45^{\mathrm{b}}$ & $6.25^{\mathrm{b}}$ & $6.89^{\mathrm{b}}$ & $1.00^{\mathrm{d}}$ & $1.00^{\mathrm{e}}$ & $1.00^{\mathrm{f}}$ & $1.00^{\mathrm{d}}$ \\
\hline & $(42.67)$ & $(54.67)$ & $(38.67)$ & $(46.67)$ & $(0.00)$ & $(0.00)$ & $(0.00)$ & $(0.00)$ \\
\hline \multirow{2}{*}{$\mathrm{T}_{10}$} & $1.00^{\mathrm{f}}$ & $1.00^{\mathrm{f}}$ & $1.00^{\mathrm{e}}$ & $1.00^{\mathrm{e}}$ & $1.00^{\mathrm{d}}$ & $1.00^{\mathrm{e}}$ & $1.00^{\mathrm{f}}$ & $1.00^{\mathrm{d}}$ \\
\hline & $(0.00)$ & $(0.00)$ & $(0.00)$ & $(0.00)$ & $(0.00)$ & $(0.00)$ & $(0.00)$ & $(0.00)$ \\
\hline $\mathrm{T}_{11}$ & $3.40^{\text {cde }}$ & $3.73^{\mathrm{de}}$ & $2.75^{\mathrm{d}}$ & $3.20^{\mathrm{d}}$ & $2.75^{\mathrm{c}}$ & $3.40^{\mathrm{cd}}$ & $2.95^{\mathrm{df}}$ & $3.40^{\mathrm{bc}}$ \\
\hline
\end{tabular}




\begin{tabular}{|c|c|c|c|c|c|c|c|c|}
\hline & $(10.67)$ & $(13.33)$ & $(6.67)$ & $(9.33)$ & $(6.67)$ & $(10.67)$ & $(8.00)$ & $(10.67)$ \\
\hline \multirow{2}{*}{$\mathrm{T}_{12}$} & $8.76^{\mathrm{a}}$ & $9.28^{\mathrm{a}}$ & $7.33^{\mathrm{a}}$ & $8.05^{\mathrm{a}}$ & $7.87^{\mathrm{a}}$ & $8.36^{\mathrm{a}}$ & $8.37^{\mathrm{a}}$ & $8.69^{\mathrm{a}}$ \\
\cline { 2 - 9 } & $(76.00)$ & $(85.33)$ & $(53.33)$ & $(64.00)$ & $(61.33)$ & $(69.33)$ & $(69.33)$ & $(74.67)$ \\
\hline $\operatorname{Lsd}_{(0.05)}$ & Sig. & Sig. & Sig. & Sig. & Sig. & Sig. & Sig. & Sig. \\
\hline
\end{tabular}

*Figures in parentheses are means of original values. Data subjected to transformation $(\sqrt{x+1})$. Treatment means with the letters in common are not significant by DNMRT test at $5 \%$ level of significance

Table 2: Dry weight of monocot and dicot weeds as influenced by various weed management practices

\begin{tabular}{|c|c|c|c|c|c|c|c|c|}
\hline \multirow{3}{*}{$\begin{array}{l}\text { Treat } \\
\text { ment }\end{array}$} & \multicolumn{4}{|c|}{ Dry weight of monocot weed $\left(\mathrm{g} / \mathrm{m}^{2}\right)$} & \multicolumn{4}{|c|}{ Dry weight of dicot weed $\left(\mathrm{g} / \mathrm{m}^{2}\right)$} \\
\hline & \multicolumn{2}{|c|}{ at 80 DAS } & \multicolumn{2}{|c|}{ at Harvest } & \multicolumn{2}{|c|}{ at 80 DAS } & \multicolumn{2}{|c|}{ at Harvest } \\
\hline & 2017-18 & 2018-19 & 2017-18 & 2018-19 & 2017-18 & 2018-19 & $2017-18$ & 2018-19 \\
\hline \multirow{2}{*}{$\mathrm{T}_{1}$} & $14.14^{\mathrm{ab}}$ & $13.01^{\mathrm{bc}}$ & $17.76^{\mathrm{ab}}$ & $16.67^{\mathrm{ab}}$ & $5.45^{\mathrm{c}}$ & $4.94^{\mathrm{bc}}$ & $5.50^{\mathrm{b}}$ & $4.17^{\mathrm{bc}}$ \\
\hline & $(201.20)$ & $(168.83)$ & $(315.67)$ & $(278.20)$ & $(29.33)$ & $(23.83)$ & $(29.63)$ & $(16.63)$ \\
\hline \multirow{2}{*}{$\mathrm{T}_{2}$} & $13.06^{\mathrm{b}}$ & $11.91^{\mathrm{c}}$ & $16.81^{\mathrm{b}}$ & $15.54^{\mathrm{b}}$ & $5.32^{\mathrm{c}}$ & $4.57^{\mathrm{c}}$ & $4.69^{\mathrm{bc}}$ & $3.78^{c}$ \\
\hline & $(172.27)$ & $(144.67)$ & $(284.20)$ & $(242.50)$ & $(27.33)$ & $(20.47)$ & $(21.47)$ & $(13.90)$ \\
\hline \multirow{2}{*}{$\mathrm{T}_{3}$} & $6.99^{c}$ & $6.52^{\mathrm{d}}$ & $3.91^{\mathrm{c}}$ & $3.12^{\mathrm{c}}$ & $4.54^{\mathrm{c}}$ & $3.99^{c}$ & $3.77^{\mathrm{c}}$ & $3.21^{\mathrm{c}}$ \\
\hline & (51.53) & (43.67) & (15.13) & $(8.77)$ & (19.77) & $(15.20)$ & (13.73) & $(9.67)$ \\
\hline \multirow{2}{*}{$\mathrm{T}_{4}$} & $8.13^{\mathrm{c}}$ & $7.28^{\mathrm{d}}$ & $4.32^{\mathrm{c}}$ & $3.69^{c}$ & $6.89^{\mathrm{b}}$ & $5.90^{\mathrm{b}}$ & $5.57^{\mathrm{b}}$ & $5.05^{\mathrm{b}}$ \\
\hline & $(66.67)$ & $(52.17)$ & $(17.87)$ & $(13.00)$ & (47.13) & $(34.60)$ & $(30.83)$ & $(24.67)$ \\
\hline \multirow{2}{*}{$\mathrm{T}_{5}$} & $7.79^{c}$ & $7.00^{\mathrm{d}}$ & $4.21^{\mathrm{c}}$ & $3.41^{\mathrm{c}}$ & $1.00^{\mathrm{d}}$ & $1.00^{\mathrm{d}}$ & $1.00^{\mathrm{d}}$ & $1.00^{\mathrm{d}}$ \\
\hline & (59.70) & (48.07) & (17.53) & $(10.70)$ & $(0.00)$ & $(0.00)$ & $(0.00)$ & $(0.00)$ \\
\hline \multirow{2}{*}{$\mathrm{T}_{6}$} & $1.00^{\mathrm{d}}$ & $1.00^{\mathrm{e}}$ & $1.00^{\mathrm{d}}$ & $1.00^{\mathrm{d}}$ & $1.00^{\mathrm{d}}$ & $1.00^{\mathrm{d}}$ & $1.00^{\mathrm{d}}$ & $1.00^{\mathrm{d}}$ \\
\hline & $(0.00)$ & $(0.00)$ & $(0.00)$ & $(0.00)$ & $(0.00)$ & $(0.00)$ & $(0.00)$ & $(0.00)$ \\
\hline \multirow{2}{*}{$\mathrm{T}_{7}$} & $5.99^{c}$ & $1.00^{\mathrm{e}}$ & $3.53^{c}$ & $1.00^{\mathrm{d}}$ & $10.44^{\mathrm{a}}$ & $11.51^{\mathrm{a}}$ & $11.83^{\mathrm{a}}$ & $12.50^{\mathrm{a}}$ \\
\hline & $(35.57)$ & $(0.00)$ & (11.53) & $(0.00)$ & (108.57) & $(131.80)$ & (138.93) & $(156.27)$ \\
\hline \multirow{2}{*}{$\mathrm{T}_{8}$} & $1.00^{\mathrm{d}}$ & $1.00^{\mathrm{e}}$ & $1.00^{\mathrm{d}}$ & $1.00^{\mathrm{d}}$ & $1.00^{\mathrm{d}}$ & $1.00^{\mathrm{d}}$ & $1.00^{\mathrm{d}}$ & $1.00^{\mathrm{d}}$ \\
\hline & $(0.00)$ & $(0.00)$ & $(0.00)$ & $(0.00)$ & $(0.00)$ & $(0.00)$ & $(0.00)$ & $(0.00)$ \\
\hline \multirow{2}{*}{$\mathrm{T}_{9}$} & $15.15^{\mathrm{ab}}$ & $14.50^{\mathrm{ab}}$ & $18.87^{\mathrm{ab}}$ & $17.27^{\mathrm{a}}$ & $1.00^{\mathrm{d}}$ & $1.00^{\mathrm{d}}$ & $1.00^{\mathrm{d}}$ & $1.00^{\mathrm{d}}$ \\
\hline & $(230.87)$ & $(212.23)$ & $(357.20)$ & (298.43) & $(0.00)$ & $(0.00)$ & $(0.00)$ & $(0.00)$ \\
\hline \multirow{2}{*}{$\mathrm{T}_{10}$} & $1.00^{\mathrm{d}}$ & $1.00^{\mathrm{e}}$ & $1.00^{\mathrm{d}}$ & $1.00^{\mathrm{d}}$ & $1.00^{\mathrm{d}}$ & $1.00^{\mathrm{d}}$ & $1.00^{\mathrm{d}}$ & $1.00^{\mathrm{d}}$ \\
\hline & $(0.00)$ & $(0.00)$ & $(0.00)$ & $(0.00)$ & $(0.00)$ & $(0.00)$ & $(0.00)$ & $(0.00)$ \\
\hline \multirow{2}{*}{$\mathrm{T}_{11}$} & $7.12^{\mathrm{c}}$ & $8.08^{\mathrm{d}}$ & $5.12^{\mathrm{c}}$ & $4.42^{\mathrm{c}}$ & $4.21^{\mathrm{c}}$ & $4.93^{\mathrm{bc}}$ & $3.76^{\mathrm{c}}$ & $4.24^{\mathrm{bc}}$ \\
\hline & (50.17) & (64.73) & $(25.60)$ & (19.33) & (16.90) & $(23.40)$ & (13.90) & $(17.50)$ \\
\hline \multirow{2}{*}{$\mathrm{T}_{12}$} & $16.53^{\mathrm{a}}$ & $15.82^{\mathrm{a}}$ & $19.24^{\mathrm{a}}$ & $18.10^{\mathrm{a}}$ & $10.63^{\mathrm{a}}$ & $12.09^{\mathrm{a}}$ & $12.43^{\mathrm{a}}$ & $13.43^{\mathrm{a}}$ \\
\hline & $(272.63)$ & $(250.37)$ & $(369.83)$ & $(326.87)$ & (113.13) & (145.97) & $(153.80)$ & $(179.70)$ \\
\hline $\operatorname{Lsd}_{(0.05)}$ & Sig. & Sig. & Sig. & Sig. & Sig. & Sig. & Sig. & Sig. \\
\hline
\end{tabular}

\section{Growth and yield attributes of wheat}

Effect of different weed management practices did not exert any significant effect on plant height recorded at 90 DAS (Table 3). Significantly higher effective tillers $/ \mathrm{m}^{2}$, total tillers $/ \mathrm{m}^{2}$ and tiller conversion index of wheat were registered under application of pre-mix herbicide sulfosulfuron + metsulfuron $(\mathrm{PoE}) 30+2 \mathrm{~g} / \mathrm{ha}$ and clodinafop + metsulfuron (PoE) $60+4 \mathrm{~g} / \mathrm{ha}$ as compared to other treatments. Higher value of tiller conversion index itself proved that better weed management practices increase the effective tillers per meter square ultimately improve the percentage of tiller conversion index. Significantly the lowest yield attributing characters viz., effective tillers $/ \mathrm{m}^{2}$, total tillers $/ \mathrm{m}^{2}$ and tiller conversion index was recorded under un-weeded control during the year of 2017-18 and 2018-19.

Post emergence application of clodinafop + metsulfuron (Premix) $60+4 \mathrm{~g} / \mathrm{ha}$ recorded significantly higher grain and straw yield as compared to rest of treatments except application of sulfosulfuron + metsulfuron (PoE) $30+2 \mathrm{~g} / \mathrm{ha}$, hand weeding at 20 and 40 DAS, pendimethalin (PE) 500 $\mathrm{g} / \mathrm{ha} f b$ sulfosulfuron (PoE) $18 \mathrm{~g} / \mathrm{ha}$, mesosulfuron + iodosulfuron $(\mathrm{PoE}) 12+2.4 \mathrm{~g} / \mathrm{ha}$ and sulfosulfuron $+2,4-\mathrm{D}$ (SS) (PoE) $25+250 \mathrm{~g} / \mathrm{ha}$. Better expression of yield achieved under said treatments could be due to effective control of weeds might have helped the crop plants to accumulate more dry matter through greater nutrient uptake that might have provided more quantity of photosynthates to developing sink in crop plants thereby produced more yield (Meena et al. 2019) ${ }^{[6]}$. The grain yield improvement by weed control with different herbicide combinations has already been reported by Bharat and Kachroo (2010) ${ }^{[3]}$, Chaudhari et al. (2017) ${ }^{[4]}$ and Patel et al. (2017) ${ }^{[9]}$.

Table 3: Growth and yield attributes of wheat as influenced by various weed management practices

\begin{tabular}{|c|c|c|c|c|c|c|c|c|c|c|c|c|}
\hline \multirow[t]{2}{*}{ Treatment } & \multicolumn{2}{|c|}{ Plant height at 90 DAS (cm } & \multicolumn{2}{|c|}{ Effective tillers $/ \mathbf{m}^{2}$} & \multicolumn{2}{|c|}{ Total tillers $/ \mathbf{m}^{2}$} & \multicolumn{2}{|c|}{ Tiller Conversion Index $(\%)$} & \multicolumn{2}{|c|}{$\begin{array}{c}\begin{array}{c}\text { Grain yield } \\
\text { (t/ha) }\end{array} \\
\end{array}$} & \multicolumn{2}{|c|}{\begin{tabular}{|c|}
$\begin{array}{c}\text { Straw yield } \\
(\mathrm{t} / \mathrm{ha})\end{array}$ \\
\end{tabular}} \\
\hline & 2017-18 & 2018-19 & 2017-18 & 2018-19 & 2017-18 & 2017-18 & 2018-19 & 2018-19 & 2017-18 & 2018-19 & 2017-18 & 2018-19 \\
\hline $\mathrm{T}_{1}$ & 85.93 & 89.93 & $238.1^{\mathrm{c}}$ & $264.0^{c}$ & $358.1^{\mathrm{de}}$ & $391.2^{\mathrm{d}}$ & $67.15^{\mathrm{cd}}$ & $67.89^{\mathrm{d}}$ & $3.63^{\mathrm{d}}$ & $3.90^{\mathrm{e}}$ & $5.84^{\mathrm{d}}$ & $6.81^{\mathrm{c}}$ \\
\hline $\mathrm{T}_{2}$ & 88.44 & 90.53 & $241.6^{c}$ & $280.0^{\mathrm{c}}$ & $366.4^{\text {cd }}$ & $408.8^{\mathrm{cd}}$ & $67.04^{\mathrm{cd}}$ & $68.47^{\mathrm{d}}$ & $3.87^{\mathrm{cd}}$ & $4.21^{\mathrm{de}}$ & $6.57^{\mathrm{bcd}}$ & $7.20^{\mathrm{bc}}$ \\
\hline $\mathrm{T}_{3}$ & 84.69 & 85.56 & $353.1^{\mathrm{a}}$ & $400.3^{\mathrm{a}}$ & $430.7^{\mathrm{abc}}$ & $480.8^{\mathrm{ab}}$ & $82.14^{\mathrm{ab}}$ & $83.55^{\mathrm{ab}}$ & $4.98^{\mathrm{ab}}$ & $5.61^{\mathrm{ab}}$ & $7.37^{\mathrm{abc}}$ & $8.40^{\mathrm{ab}}$ \\
\hline $\mathrm{T}_{4}$ & 86.03 & 88.77 & $277.1^{\mathrm{bc}}$ & $311.2^{\mathrm{bc}}$ & $387.2^{\mathrm{abcd}}$ & $426.7^{\mathrm{abcd}}$ & $71.53^{\mathrm{abcd}}$ & $73.35^{\mathrm{abcd}}$ & $4.37^{\mathrm{bcd}}$ & $4.96^{\mathrm{bcd}}$ & $6.89^{\mathrm{abcd}}$ & $7.59^{\mathrm{abc}}$ \\
\hline $\mathrm{T}_{5}$ & 83.93 & 86.90 & $337.3^{\mathrm{a}}$ & $373.9^{\mathrm{ab}}$ & $419.5^{\mathrm{abcd}}$ & $471.5^{\mathrm{abc}}$ & $80.30^{\mathrm{abc}}$ & $79.22^{\text {abcd }}$ & $4.73^{\mathrm{abc}}$ & $5.36^{\mathrm{abc}}$ & $7.08^{\mathrm{abcd}}$ & $8.22^{\mathrm{abc}}$ \\
\hline $\mathrm{T}_{6}$ & 84.10 & 88.96 & $365.9^{\mathrm{a}}$ & $413.6^{\mathrm{a}}$ & $448.3^{\mathrm{a}}$ & $482.7^{\mathrm{ab}}$ & $81.53^{\mathrm{ab}}$ & $85.77^{\mathrm{a}}$ & $5.41^{\mathrm{a}}$ & $5.93^{\mathrm{ab}}$ & $8.02^{\mathrm{a}}$ & $8.84^{\mathrm{a}}$ \\
\hline $\mathrm{T}_{7}$ & 87.81 & 90.74 & $260.8^{\mathrm{c}}$ & $296.0^{c}$ & $380.0^{\mathrm{bcd}}$ & $422.9^{\mathrm{bcd}}$ & $68.63^{\mathrm{cd}}$ & $70.10^{\mathrm{bcd}}$ & $4.29^{\mathrm{bcd}}$ & $4.60^{\text {cde }}$ & $6.83^{\mathrm{bcd}}$ & $7.50^{\mathrm{bc}}$ \\
\hline $\mathrm{T}_{8}$ & 85.75 & 87.98 & $367.2^{\mathrm{a}}$ & $426.4^{\mathrm{a}}$ & $440.3^{\mathrm{ab}}$ & $493.6^{\mathrm{a}}$ & $83.31^{\mathrm{a}}$ & $86.28^{\mathrm{a}}$ & $5.47^{\mathrm{a}}$ & $6.12^{\mathrm{a}}$ & $8.15^{\mathrm{a}}$ & $8.95^{\mathrm{a}}$ \\
\hline
\end{tabular}




\begin{tabular}{|c|c|c|c|c|c|c|c|c|c|c|c|c|}
\hline $\mathrm{T}_{9}$ & 88.47 & 89.27 & $248.8^{\mathrm{c}}$ & $291.5^{\mathrm{c}}$ & $369.1^{\mathrm{cd}}$ & $419.7^{\mathrm{bcd}}$ & $67.56^{\mathrm{cd}}$ & $69.65^{\mathrm{cd}}$ & $4.26^{\mathrm{bcd}}$ & $4.53^{\text {cde }}$ & $6.40^{\mathrm{cd}}$ & $7.21^{\mathrm{bc}}$ \\
\hline $\mathrm{T}_{10}$ & 81.58 & 82.96 & $328.8^{\mathrm{ab}}$ & $372.5^{\mathrm{ab}}$ & $422.1^{\mathrm{abc}}$ & $465.6^{\mathrm{abc}}$ & $78.03^{\mathrm{abc}}$ & $79.78^{\mathrm{abcd}}$ & $4.75^{\mathrm{abc}}$ & $5.46^{\mathrm{abc}}$ & $7.30^{\mathrm{abc}}$ & $8.65^{\mathrm{ab}}$ \\
\hline $\mathrm{T}_{11}$ & 90.04 & 91.87 & $341.6^{\mathrm{a}}$ & $390.9^{a}$ & $428.8^{\mathrm{abc}}$ & $475.7^{\mathrm{abc}}$ & $79.82^{\mathrm{abc}}$ & $82.27^{\mathrm{abc}}$ & $5.30^{\mathrm{a}}$ & $5.97^{\mathrm{a}}$ & $7.91^{\mathrm{ab}}$ & $8.86^{\mathrm{a}}$ \\
\hline $\mathrm{T}_{12}$ & 91.13 & 93.48 & $176.8^{\mathrm{d}}$ & $208.8^{\mathrm{d}}$ & $304.3^{\mathrm{e}}$ & $378.4^{\mathrm{d}}$ & $58.41^{\mathrm{d}}$ & $55.64^{\mathrm{e}}$ & $1.65^{\mathrm{e}}$ & $1.90^{\mathrm{f}}$ & $3.52^{\mathrm{e}}$ & $4.54^{\mathrm{d}}$ \\
\hline $\operatorname{Lsd}_{(0.05)}$ & NS & NS & Sig. & Sig. & Sig. & Sig. & Sig. & Sig. & Sig. & Sig. & Sig. & Sig. \\
\hline
\end{tabular}

*Mean followed by common letter (s) in column are not significant by DNMRT test at 5\% level of significance

\section{Nutrient uptake by weeds}

Result in respect of nutrient uptake by weed was significantly affected by various weed management practices (Table 4). Significantly lower nitrogen, phosphorus and potash uptake by weeds wrere recorded under application of pendimethalin (PE) $500 \mathrm{~g} / \mathrm{ha} f b$ sulfosulfuron (PoE) $18 \mathrm{~g} / \mathrm{ha}$ than other treatments except twice hand weeding at 20 and 40 DAS, application of sulfosulfuron + 2,4-D SS (PoE) $25+250 \mathrm{~g} / \mathrm{ha}$ and sulfosulfuron (PoE) $25 \mathrm{~g} / \mathrm{ha}$ during both the years of experimentation. Pre-mix herbicide formulation provided cent per cent of weed control so, it may helpful to eliminate the crop-weed competition from early phase for nutrient uptake over control. Similarly, result was reported by Meena et al. (2016) ${ }^{[7]}$. Sole application of metsulfuron methyl (PoE) 4 $\mathrm{g} / \mathrm{ha}$ ) and clodinafop (PoE) $60 \mathrm{~g} / \mathrm{ha}$ recorded comparative higher nutrient (NPK) uptake by weed than the rest of treatment except un-weeded control during the year of 201718 and 2018-19.

Table 4: Nutrient uptake by weed as influenced by various weed management practices

\begin{tabular}{|c|c|c|c|c|c|c|}
\hline \multirow{3}{*}{ Treatment } & \multicolumn{6}{|c|}{ Nutrient uptake by weed(kg/ha) at harvest } \\
\cline { 2 - 7 } & \multicolumn{2}{|c|}{ N uptake } & \multicolumn{2}{c|}{ P uptake } & \multicolumn{2}{c|}{ K uptake } \\
\cline { 2 - 7 } & $\mathbf{2 0 1 7 - 1 8}$ & $\mathbf{2 0 1 8 - 1 9}$ & $\mathbf{2 0 1 7 - 1 8}$ & $\mathbf{2 0 1 8 - 1 9}$ & $\mathbf{2 0 1 7 - 1 8}$ & $\mathbf{2 0 1 8 - 1 9}$ \\
\hline $\mathrm{T}_{1}$ & $6.07^{\mathrm{b}}$ & $4.46^{\mathrm{ab}}$ & $1.47^{\mathrm{b}}$ & $1.15^{\mathrm{b}}$ & $7.24^{\mathrm{b}}$ & $5.46^{\mathrm{b}}$ \\
\hline $\mathrm{T}_{2}$ & $5.74^{\mathrm{b}}$ & $4.29^{\mathrm{ab}}$ & $1.30^{\mathrm{b}}$ & $1.06^{\mathrm{c}}$ & $6.08^{\mathrm{c}}$ & $5.19^{\mathrm{b}}$ \\
\hline $\mathrm{T}_{3}$ & $1.01^{\mathrm{d}}$ & $0.86^{\mathrm{c}}$ & $0.22^{\mathrm{f}}$ & $0.18^{\mathrm{f}}$ & $1.12^{\mathrm{e}}$ & $0.98^{\mathrm{e}}$ \\
\hline $\mathrm{T}_{4}$ & $1.32^{\mathrm{d}}$ & $1.13^{\mathrm{c}}$ & $0.34^{\mathrm{e}}$ & $0.27^{\mathrm{e}}$ & $1.48^{\mathrm{e}}$ & $1.37^{\mathrm{d}}$ \\
\hline $\mathrm{T}_{5}$ & $1.10^{\mathrm{d}}$ & $0.91^{\mathrm{c}}$ & $0.28^{\mathrm{ef}}$ & $0.21^{\mathrm{ef}}$ & $1.21^{\mathrm{e}}$ & $1.15^{\mathrm{de}}$ \\
\hline $\mathrm{T}_{6}$ & $0.00^{\mathrm{e}}$ & $0.00^{\mathrm{d}}$ & $0.00^{\mathrm{g}}$ & $0.00^{\mathrm{g}}$ & $0.00^{\mathrm{f}}$ & $0.00^{\mathrm{f}}$ \\
\hline $\mathrm{T}_{7}$ & $3.31^{\mathrm{c}}$ & $2.58^{\mathrm{b}}$ & $0.68^{\mathrm{d}}$ & $0.56^{\mathrm{d}}$ & $3.64^{\mathrm{d}}$ & $3.77^{\mathrm{c}}$ \\
\hline $\mathrm{T}_{8}$ & $0.00^{\mathrm{e}}$ & $0.00^{\mathrm{d}}$ & $0.00^{\mathrm{g}}$ & $0.00^{\mathrm{g}}$ & $0.00^{\mathrm{f}}$ & $0.00^{\mathrm{f}}$ \\
\hline $\mathrm{T}_{9}$ & $5.93^{\mathrm{b}}$ & $4.49^{\mathrm{ab}}$ & $1.36^{\mathrm{b}}$ & $1.01^{\mathrm{c}}$ & $7.12^{\mathrm{b}}$ & $5.38^{\mathrm{b}}$ \\
\hline $\mathrm{T}_{10}$ & $0.00^{\mathrm{e}}$ & $0.00^{\mathrm{d}}$ & $0.00^{\mathrm{g}}$ & $0.00^{\mathrm{g}}$ & $0.00^{\mathrm{f}}$ & $0.00^{\mathrm{f}}$ \\
\hline $\mathrm{T}_{11}$ & $1.18^{\mathrm{d}}$ & $1.04^{\mathrm{c}}$ & $0.28^{\mathrm{ef}}$ & $0.23^{\mathrm{ef}}$ & $1.29^{\mathrm{e}}$ & $1.25^{\mathrm{de}}$ \\
\hline $\mathrm{T}_{12}$ & $14.49^{\mathrm{a}}$ & $11.26^{\mathrm{a}}$ & $2.29^{\mathrm{a}}$ & $1.71^{\mathrm{a}}$ & $15.68^{\mathrm{a}}$ & $14.07^{\mathrm{a}}$ \\
\hline $\mathrm{Lsd}_{(0.05)}$ & Sig. & Sig. & Sig. & Sig. & Sig. & Sig. \\
\hline
\end{tabular}

* Mean followed by common letter (s) in column is not significant by DNMRT test at $5 \%$ level of significance

\section{Reference}

1. Angiras NN, Kumar S, Rana SS, Sharma N. Standardization of dose and time of application of clodinafop-propargyl to manage weeds in wheat. Himachal Journal of Agricultural Research. 2008; 34(2):15-18.

2. Anonymous. Agricultural statistics at a glance 2018, Department of Agriculture Cooperation \& Farmers Welfare, Government of India, KrishiBhawan, New Delhi, 2018. http://www.agricoop.nic.in.

3. Bharat, $\mathrm{R}$ and Kachroo, D. Bio-efficacy of herbicides on weeds in wheat (Triticum aestivum L.) and its residual effect on succeeding cucumber (Cucumis sativus L.). Indian Journal of Agronomy. 2010; 55(1):46-50.

4. Chaudhari DD, Patel VJ, Patel HK, Aakask Mishra, Patel BD, Patel RB. Assessment of pre-mix broad spectrum herbicides for weed management in wheat. Indian Journal of Weed Science. 2017; 49(1):33-35.

5. Lekhchand, Puniya R. Bio-efficacy of alone and mixture of herbicides against complex weed flora in wheat (Triticum aestivum) under sub-tropical conditions. Indian Journal of Agricultural Science. 2017; 87(9):1149-1154.

6. Meena V, Kaushik ML, Dotaniya, Meena BP, Das H. Bio-efficacy of readi-mix herbicides on weeds and productivity in late-sown wheat. Indian Journal of Weed Science. 2019; 51(4):344-351.

7. Meena OP, Nepalia V, Singh D, Verma A, Choudhary R. Herbicide combinations for broad spectrum weed control in wheat. Indian Journal of Weed Science. 2016; 48(3):325-327.
8. Malik RS, Yadav A, Kumari R. Ready-mix formulation of clodinafop-propargyl + metsulfuron-methyl against complex weed flora in wheat. Indian Journal of Weed Science. 2013; 45(3):179-182.

9. Patel BD, Chaudhari DD, Patel VJ, Patel HK, Aakask Mishra, Parmar DJ. Influence of broad spectrum herbicides on yield and complex weed flora of wheat (Triticum aestivum L.). Research on Crops. 2017; 18(3):433-437.

10. Punia SS, Yadav D, Yadav A, Malik RS, Malik YP. Bioefficiency and phytotoxicity of herbicides UPH-206 (clodinafop propargyl 15\% + metsulfuron 1\%) for the control of complex weed flora in wheat and its residual effect on succeeding sorghum crop. Indian Journal of Weed Science. 2008; 40(3\&4):176-179. 